\title{
AN IMPROVED EXEMPLAR-BASED IMAGE INPAINTING ALGORITHM FOR ERROR CONCEALMENT
}

\author{
Hwang Byongsu, Jo Jonghyon and Ri Cholsu \\ Institute of Information Science, Kim Il-sung University, Democratic People's Republic of Korea
}

\begin{abstract}
Error concealment is an important technique to improve the video quality in case that the video frame is corrupted during transmission. A spatial error concealment algorithm based on the improved exemplar-based image inpainting is presented. Each corrupted macroblock is separated into sixteen $4 \times 4$ blocks, and a $4 \times 4$ block-based image inpainting is used for error concealment. In the exemplar-based inpainting process the best matching patch is determined by calculating the weighted sum of squared differences for the available patch regions which includes already concealed blocks. Experimental results show that our proposed method achieves better quality in terms of objective and subjective evaluations compared with the previous algorithms.
\end{abstract}

Keywords:

Video Communication, Error Concealment, Exemplar Based Image Inpainting

\section{INTRODUCTION}

When the compressed video frames are transmitted over errorprone channels, the packet losses lead to the corrupted region in frame. Video error concealment is a technique to recover the corrupted regions in frame. Error concealment (EC) techniques can be categorized into two classes: spatial error concealment (SEC) and temporal error concealment (TEC). To recover corrupted block, SEC exploits correctly received block information within current frame, while TEC utilizes temporal correlations in a video sequence.

This work focuses on spatial error concealment. A spatial error concealment's interpolation algorithm utilizes spatial correlations in image. It has low computational complexity and is useful for real-time processing. Interpolation algorithms reconstruct the missing macroblocks by using simple bilinear interpolation (BI) from four pixels in surrounding macroblocks [1], or using complex directional interpolation techniques based on estimation of edge directions $[2,4]$.

Wang et al. proposed the best neighbour matching (BNM) method which reconstructs the lost blocks by using blockwise similarity measurement within the image [5]. The non-uniform rational B-spline (NURBS) interpolation and the block matching interpolation proposed by Pak et al. and Hsia et al. are available for consecutive block loss [6,7]. And also other concealment techniques such as orientation adaptive interpolation, content adaptive concealment and model adaptive concealment method have been employed $[8,9,10]$.

$\mathrm{Li}$ et al. proposed the SEC strategy which selects the appropriate spatial method by location information and Intra Mode of neighboring received MBs [19]. Lin T. L. et al. solved the optimization problem by the primal-dual interior point method, and proposed an application of sparse optimization in the error concealment area [20].
Recently, error concealment algorithms using image inpainting have been widely studied.

Image inpainting was originally developed for the purpose of editing image to fill specific regions $[11,14]$. Criminisi et al. proposed an exemplar-based image inpainting method, which first decides the filling order based on the structure information, searches the best matching patch within surrounding image, and then fills the missing region by copying the found patch $[11,12]$. $\mathrm{Li}$ et al. proposed an improved exemplar-based image inpainting technique to depress the growth of corrupted image content [13].

Ebdelli et al. pointed out that similarity metric is the main parameter which influences searching the best matching patch in the uncorrupted region during image inpainting process [16]. Although the sum of squared differences (SSD) is the most widely used similarity metric for patches matching, the SSD is suitable for the copy of pixels from uniform regions. Hence several variants of metric, such as SSD metric based on Bhattacharya distance, was proposed to improve the inpainting quality.

Many works were devoted to employ image inpainting for error concealment. Although image inpainting can be used for concealing corrupted regions, it is not suitable for real-time applications because of its high computational complexity.

To overcome the defect of image inpainting, Chen et al. proposed a new hardware-oriented image inpainting algorithm for I-frame error concealment [14], and Guan et al. presented the modification of the exemplar-based image inpainting algorithm suitable for hardware implementation to design a hardware system architecture for an image inpainting-based error concealment [15]. Ebdelli et al. proposed an error concealment algorithm combining motion interpolation and exampler-based video inpainting [17]. The algorithm first estimates the motion information of the lost blocks in the video sequence using a method called BMFI (Bilinear Motion Field Interpolation). Then, the video inpainting algorithm approximates the missing texture as a linear combination of the most similar blocks. A hybrid interframe error concealment algorithm using improved image inpainting was proposed in [18]. Here, Hou et al. presented an improved exemplar-based image inpainting algorithm with modified confidence function, isophote function and repairing direction.

In this paper, we propose an error concealment algorithm which generates a layer composing the available patch region that includes already concealed blocks by implementing a $4 \times 4$ blockbased inpainting process.

We also propose a weighted SSD calculation method, where distance is calculated as the SSD multiplied by different weight depending on whether pixel is correctly received one or concealed one. Additionally, the proposed method is compared with the previous method. 


\section{PROPOSED EXEMPLAR-BASED IMAGE INPAINTING ALGORITHM FOR SPATIAL ERROR CONCEALMENT}

\subsection{EXEMPLAR-BASED IMAGE INPAINTING TECHNIQUE}

Exemplar-based image inpainting technique calculates the priority value of pixels to determine inpainting order of the corrupted region, and searches the best inpainting block for the pixel with the highest priority value inside uncorrupted region $[11,12]$. The best inpainting block is the most similar region to the corrupted region, namely, the region which has the smallest sum of square differences (SSD). Inpainting process is commonly carried out towards interior from the outermost boundaries until all corrupted pixels are concealed. Exemplar-Based inpainting approach is shown in Fig.1.

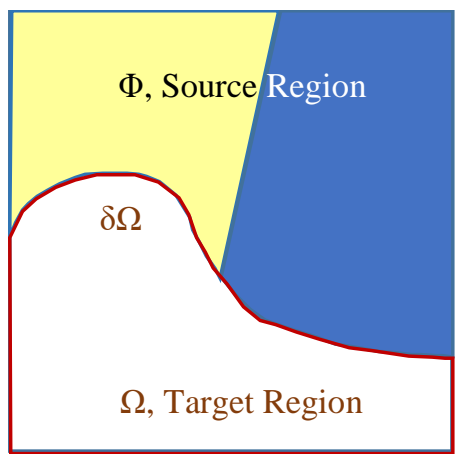

(a)

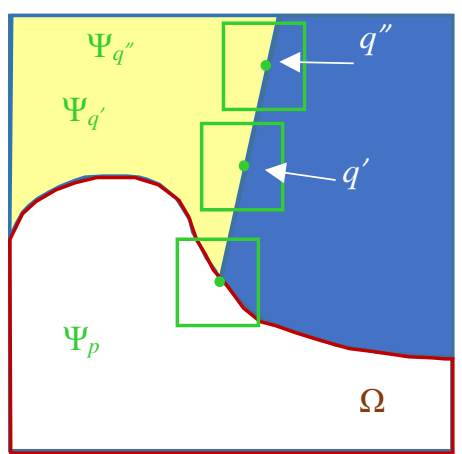

(c)

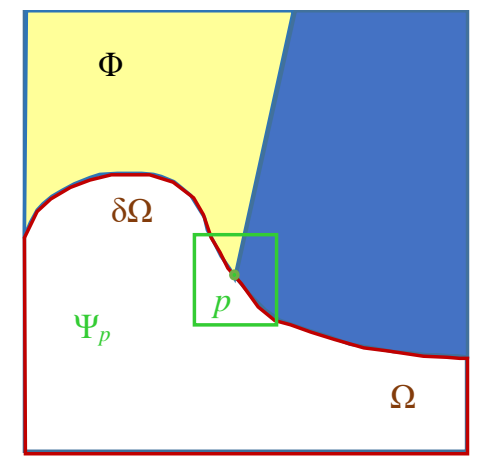

(b)

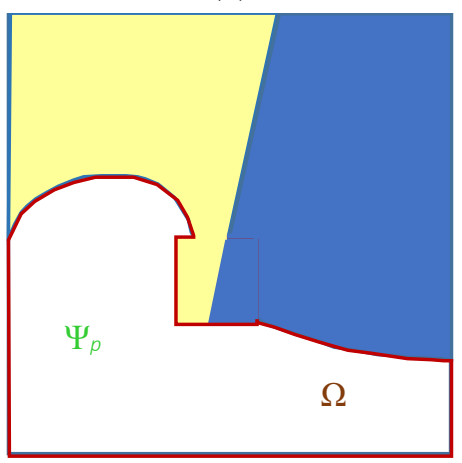

(d)
Fig.1. Examplar-Based inpainting approach [11] (a) Input image with the missing region $(\Omega)$ (b) Computation of the highest priority patch $\left(\Psi_{p}\right)(\mathrm{c})$ Search of the best matching patch $\left(\Psi_{q}\right)$ to $\Psi_{p}$ in the source region $(\Phi)(\mathrm{d})$ Input image after inpainting a patch $\Psi_{p}$ using $\Psi_{q}$. (Illustration is taken from [11])

As shown in Fig.1, $\Omega$ is the region of corrupted macroblocks, $\partial \Omega$ is the contour of $\Omega$, and $\Phi$ is the region of correct macroblocks. Firstly, priority $P(p)$ is calculated for each pixel $p$ on $\partial \Omega$ as follows:

$$
P(p)=C(p) \times D(p)
$$

where $C(p)$, the confidence term of pixel p, is defined as follows:

$$
C(p)=\frac{\sum_{q \in \Psi_{p} \cap(1-\Omega)} C(q)}{\left|\Psi_{p}\right|}
$$

where, $\Psi_{p}$ is the patch region containing pixel $p$, which is commonly formed using the pixel $p$ as the center of a $3 \times 3$ rectangular region. $\left|\Psi_{p}\right|$ is the count of pixels within $\Psi_{p . q} q$ is any pixel in the image, $C(q)$ is 1 if $q$ is in $\Phi, C(q)$ is 0 if $q$ is in $\Omega . D(p)$ is defined as follows:

$$
D(p)=\frac{\left|\nabla I_{p} \perp n_{p}\right|}{\alpha}
$$

where $n_{p}$ is a unit vector orthogonal to the fill front $\partial \Omega$ centered at $p$ and $\alpha$ is a normalizing constant $(\alpha=255) . \mathrm{D}(\mathrm{p})$ is directly proportional to the gradient strength.

In Fig.1, for example, we can assume that $\Psi_{p}$, patch of pixel $p$ on $\partial \Omega$, has the highest priority since it contains a strong edge. Then, the inpainting process starts from the patch with the highest priority value, and finds the best matching patch inside given search range. Here, the best matching patch means the most similar one.

The best matching patch can be determined by follows:

$$
\begin{gathered}
\Psi_{\bar{q}}=\arg \min _{\Psi_{\hat{q}} \in \Phi} d\left(\Psi_{\hat{p}}, \Psi_{\hat{q}}\right) \\
\Psi_{\hat{p}}=\Psi_{p} \cap \Phi
\end{gathered}
$$

where, $\Psi_{\hat{p}}$ is the intersection of patch $\Psi_{p}$ and $\Phi, \Psi_{\hat{q}}$ is the region inside $\Psi_{q}$, which is the same shape as $\Psi_{\hat{p}} . d($.$) is the distance$ between two patches, which is commonly defined as the sum of square difference of the available pixels inside two patches.

Finally, the patch $\Psi_{p}$ is filled in with the best matching patch in the source region $(\Phi)$. The same procedure is iterated until all corrupted pixels are concealed.

In [15], Guan et al. proposed $4 \times 4$ block-based image inpainting. Each corrupted macroblock is separated into sixteen $4 \times 4$ blocks. The patch size is set to a $12 \times 12$ pixel region which includes one center $4 \times 4$ block and eight $4 \times 4$ neighbouring blocks. The region inside each $12 \times 12$ area is repaired by using the same candidates.

The $4 \times 4$ block with the highest priority $P(p)$, which is calculated as the confidence term multiplied by the data term, will be selected as the first to be processed. In order to retain the edge continuity inside the macroblock, data term $D(p)$ is defined as the number of extended edge points inside the $4 \times 4$ block. After searching the best matching patch within the search range, the corrupted region of the $12 \times 12$ patch is concealed.

In [18], Hou et al. presented an improved $4 \times 4$ block-based image inpainting method for error concealment. Presented method improves $4 \times 4$ block-based image inpainting algorithm modifying confidence function, isophote function and repairing direction. This paper focuses on improving the method presented in [18].

\subsection{PROPOSED EXEMPLAR-BASED IMAGE INPAINTING ALGORITHM FOR ERROR CONCEALMENT}

\subsubsection{Extension of Available Patch Region:}

In [18], each corrupted macroblock is separated into sixteen $4 \times 4$ blocks, priority of each block is calculated according to the 
status of surrounding blocks, and the $4 \times 4$ block with the highest priority is selected as the first one to be inpainted.

Consecutively, presented algorithm searches the matching block with the smallest SSD, and replaces the corrupted block by copying pixels in the best matching block. The patch size is set to a $12 \times 12$ pixel region which includes one center $4 \times 4$ block and eight $4 \times 4$ neighboring blocks. In each inpainting procedure for the patch region, only one center $4 \times 4$ block is recovered, and another blocks in the patch region are not recovered.

Moreover, in order to lower computational complexity, presented algorithm determines priority order calculating confidence and isophote only once, and carries out the whole inpainting process for a layer according to this priority order.

The Fig. 2 shows illustration of blocks in a $4 \times 4$ block-based image inpainting. Where current block is the center $4 \times 4$ block to be inpainted, red framework is the available patch region to be used in searching the best inpainting block.

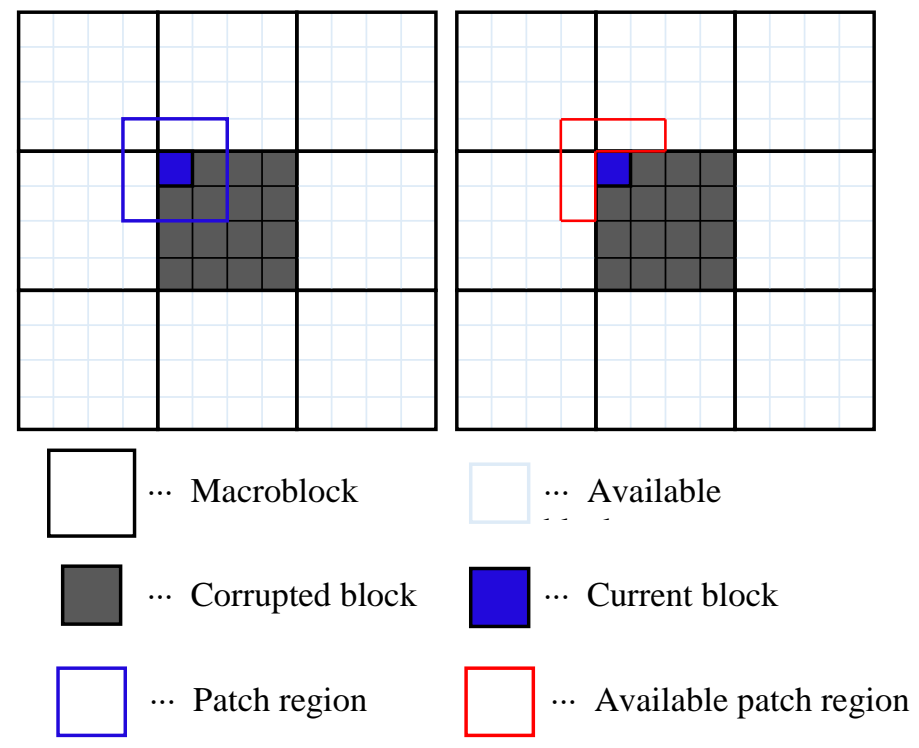

Fig.2. Illustration of blocks in $4 \times 4$ block-based inpainting

Above mentioned available patch region is just $\Psi_{\hat{p}}$ region in Eq.(5). The Fig.3 shows extension method of available patch region presented in this paper. Where light grey region are the blocks already concealed in the previous inpainting procedures. As shown in Fig.3(a), EC algorithm presented in [18] carries out the whole inpainting process for a layer composing $\Psi_{\hat{p}}$ without already concealed blocks.

In contrast with above, we present the method which carries out the inpainting process for a layer composing $\Psi_{\hat{p}}$ including already concealed blocks, as shown Fig.3(b). This is because already recovered blocks have higher priority than current block in the inpainting process for a layer, in other words, those recovered blocks have more additional information such as edge information.

Therefore, composing available patch region with already concealed blocks results in improving the correctness of searching the best matching.
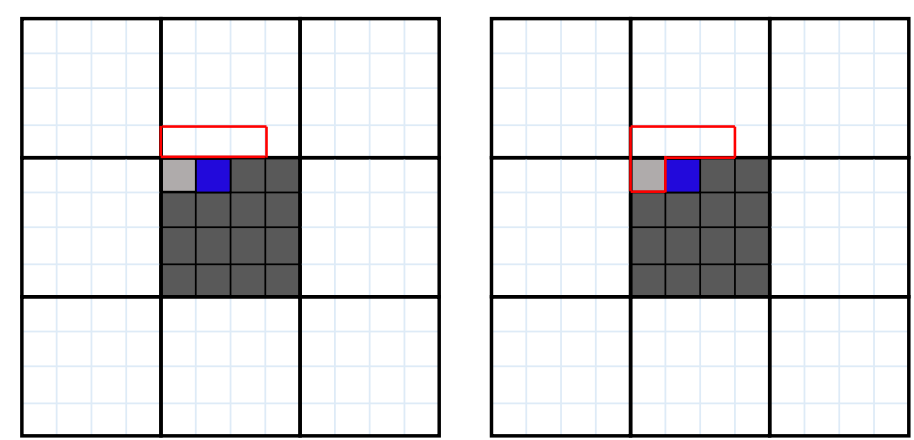

Fig.3. Extension of available patch region. (a) Available patch region without already concealed blocks (b) Available patch region including the concealed blocks

\subsubsection{Calculation of Weighted SSD:}

Ebdelli et al. pointed out that similarity metric is the main parameter which influences searching the best matching patch during image inpainting process [16]. The sum of squared differences (SSD) is the most widely used similarity metric for patches matching. In order to search the best matching patch, we first search the available candidate patch region $\Psi_{\hat{p}}$ which is the same shape as $\Psi_{\hat{p}}$ discussed in above section, and then calculate SSD between two available patch regions using Eq.(4). The same procedure is applied iteratively within the limited search range. Finally, we set the candidate patch with the smallest SSD as the best matching patch.

Here, Note that confidence of correctly received pixel is different from concealed one during calculation of SSD. Hence this paper proposes a weighted SSD calculation method, where distance is calculated as the SSD multiplied by different weight depending on whether pixel is correct one or concealed one while calculating distance.

The Fig.4 shows the placements of the available patch region $\Psi_{\hat{p}}$ and the available candidate patch region $\Psi_{\hat{q}}$ during distance calculation. Where, light blue region is a $4 \times 4$ candidate inpainting block, and green framework is the available candidate patch region $\Psi_{\hat{q}}$ to be used for calculating SSD. During SSD calculation, as shown in Fig.4, or pixel inside $\Psi_{\hat{p}}$ can be in the concealed region, or pixel inside $\Psi_{\hat{q}}$ can be in the concealed region. Given two available patch regions $\Psi_{\hat{p}}$ and $\Psi_{\hat{q}}$, the weighted SSD metric is defined as:

$$
\begin{gathered}
d_{S S D}\left(\Psi_{\hat{p}}, \Psi_{\hat{q}}\right)=\sum_{p_{k} \in \Psi_{\hat{p}}} w_{p k} \cdot w_{q k} \cdot\left(C_{p k}-C_{q k}\right)^{2} \\
w_{p k}=\left\{\begin{array}{cc}
1 & p_{k} \in \text { correct region } \\
w_{c} & p_{k} \in \text { concealed region }
\end{array}\right. \\
w_{q k}=\left\{\begin{array}{cc}
1 & q_{k} \in \text { correct region } \\
w_{c} & q_{k} \in \text { concealed region }
\end{array}\right.
\end{gathered}
$$

where $p_{k}$ is each pixel inside $\Psi_{\hat{p}}, q_{k}$ is pixel at the same position as $p_{k}$ inside $\Psi_{\hat{p}}, w_{p k}$ is weighting coefficient relative to pixel $p_{k}$, $w_{q k}$ is weighting coefficient relative to pixel $q_{k}$, and $C_{p}$ is the value of pixel $p . w_{c}$ is set to constant value between 0 and 1 . 


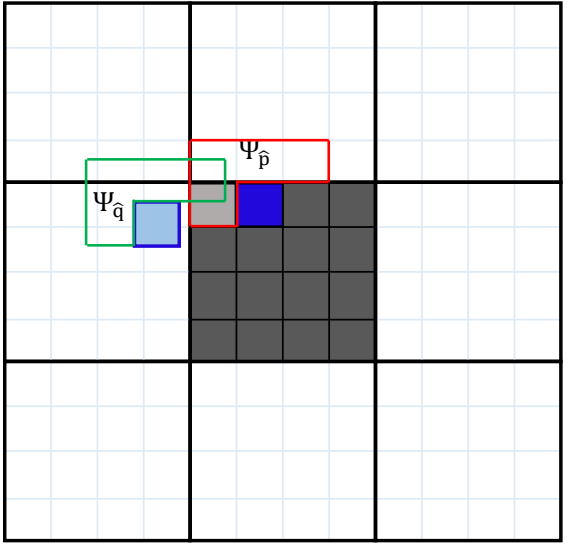

$\square$... Current block $\square$... Candidate block

… Available candidate Patch region

Fig.4. Placement of two available patch regions $\Psi_{\hat{p}}$ and $\Psi_{\hat{q}}$ during distance calculation

Thus, the weighted SSD is defined as Eq.(6) so that already concealed regions are also referred while the correctly received pixels have definitive contribution for determining the best matching patch. As a result, correctness of error concealment is certainly improved. The algorithm 1 describes the proposed error concealment algorithm, which includes a pre-processing step followed by the proposed inpainting algorithm.

\section{Algorithm 1. Proposed EC Algorithm}

Input: A corrupted image.

Output: An error concealed image.

1: Separate corrupted region into corrupted MBs

2: for each corrupted MB in the corrupted region do // Proposed exemplar-based image inpainting (Start)

3: $\quad$ Separate corrupted MB into sixteen $4 \times 4$ blocks

4: $\quad$ Calculate priority of each $4 \times 4$ block using Eq.(1)

5: while unrecovered block exist in corrupted MB do

6: $\quad$ Select the $4 \times 4$ block with the highest priority

7: $\quad$ Search the matching block with the smallest weighted SSD using Eq.(6)

8: $\quad$ Recover one center $4 \times 4$ block using the best matching block

9: $\quad$ end while

// Proposed exemplar-based image inpainting (End)

10: end for

\section{EXPERIMENTAL RESULTS}

To evaluate the performance of the proposed algorithm, a variety of experiments are conducted. We compare performance with other state-of-the-art exemplar-based inpainting method [18]. Peak signal-to-noise ratio (PSNR) for the whole image is used as the objective measurement in the experiments.
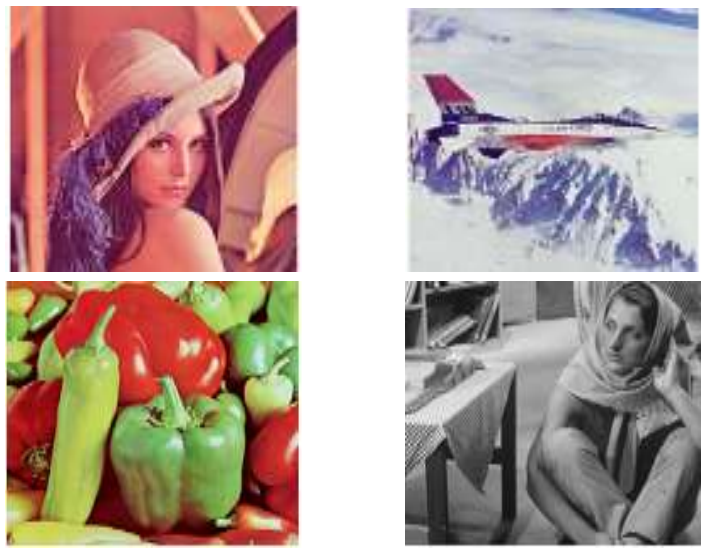

Fig.5. Original test images
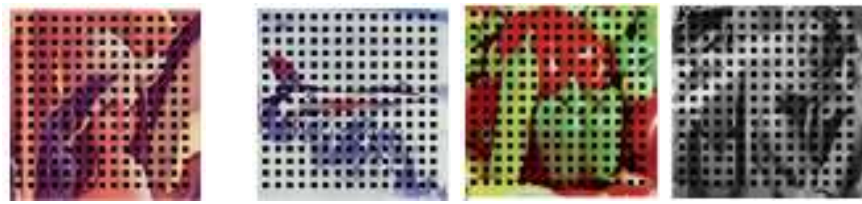

(a) Error image
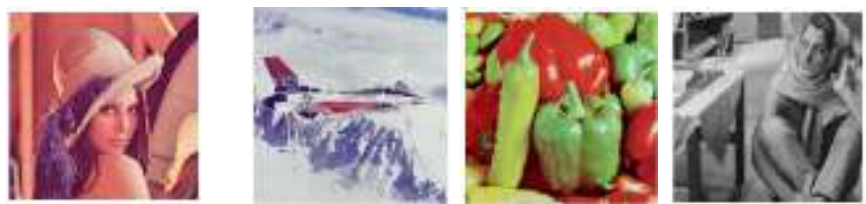

(b) Previous EC [18]
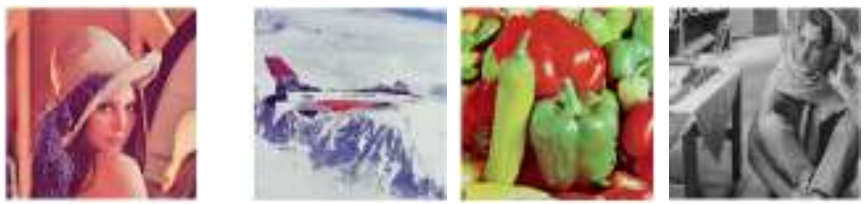

(c) Proposed

Fig.6. Subjective comparison between the proposed EC algorithm and previous work for $16 \times 16$ regular isolated loss

The original test images are shown in Fig.5, including Lena $(512 \times 512)$, F16 $(512 \times 512)$, Peppers $(512 \times 512)$, and Barbara $(512 \times 512)$.

In our experiments, we have set $w_{c}$ to 0.85 . The experiments has been carried out on images involving $16 \times 16$ regular isolated loss $(\approx 25 \%$ loss$)$. The PSNR comparison results of recovered images are given in Table.1.

Table.1. Performance comparison in PSNR for $16 \times 16$ regular isolated loss

\begin{tabular}{|c|c|c|}
\hline Image & Previous Method [18] & Proposed Method \\
\hline Lena & 23.11 & 23.17 \\
\hline F16 & 21.45 & 21.61 \\
\hline Peppers & 23.44 & 23.57 \\
\hline Barbara & 17.79 & 17.87 \\
\hline Average & $\mathbf{2 1 . 4 5}$ & $\mathbf{2 1 . 5 6}$ \\
\hline
\end{tabular}


As can be observed from the table, the proposed algorithm achieves the better performance than the previous method [18]. In particular, when compared with the recently proposed method [18], our method shows an average PSNR improvement of $0.11 \mathrm{~dB}$, obtains a maximum gain of $0.16 \mathrm{~dB}$ in terms of PSNR. Subjective quality comparisons are given in Fig.6.

Subjective comparison shows that our method gives a better reconstruction quality compared to the previous method [18]. As can be seen from objective and subjective evaluations, our method takes obvious improvements over other error concealment algorithms which uses exemplar-based image inpainting.

\section{CONCLUSION}

In this paper, we proposed an error concealment algorithm which uses improved exemplar-based image inpainting. To improve perceptual quality of recovery, $4 \times 4$ block-based image inpainting is carried out to conceal the corrupted macro-blocks. In order to determine the best matching patch in exemplar-based inpainting, the weighted SSD is calculated for the available patch regions which also includes already concealed blocks in the previous inpainting procedures. We compare our method with the recently proposed EC algorithm based on image inpainting [18]. Experimental results show that the proposed method takes noticeable improvements in performance for $16 \times 16$ regular isolated loss on objective and subjective measures.

\section{REFERENCES}

[1] S. Aign and K. Fazel, "Temporal and Spatial Error Concealment Techniques for Hierarchical MPEG-2 Video Codec", Proceedings of IEEE International Conference Communication, pp. 1778-1783, 1995.

[2] W. Kwok and H. Sun, "Multi-Directional Interpolation for Spatial Error Concealment", IEEE Transactions on Consumer Electronics, Vol. 39, No. 3, pp. 455-460, 1993.

[3] Jae-Won Suh and Yo-Sung Ho, "Error Concealment based on Directional Interpolation", IEEE Transactions on Consumer Electronics, Vol. 43, No. 3, pp. 295-302, 1997.

[4] Wonki Kim, Jasung Koo and Jechang Jeong, "Fine Directional Interpolation for Spatial Error Concealment", IEEE Transactions on Consumer Electronics, Vol. 52, No. 3, pp. 1050-1056, 2006.

[5] Z. Wang, Y. Yu and D. Zhang, "Best Neighborhood Matching: An Information Loss Restoration Technique for Block based Image Coding Systems", IEEE Transactions on Image Processing, Vol. 7, No. 6, pp. 1056-1061, 1998.

[6] J.W. Park and S.U. Lee, "Recovery of Corrupted Image Data based on the NURBS Interpolation", IEEE Transactions on Circuits and Systems for Video Technology, Vol. 9, No. 10, pp. 1003-1008, 1999.

[7] S.C. Hsia, "An Edge-Oriented Spatial Interpolation for Consecutive Block Error Concealment", IEEE Signal Processing Letters, Vol. 11, No. 6, pp. 577-580, 2004.
[8] X. Li and M. Orchard, "Novel Sequential ErrorConcealment Techniques using Orientation Adaptive Interpolation", IEEE Transactions on Circuits and Systems for Video Technology, Vol. 12, No. 10, pp. 857-864, 2002.

[9] Zhang Rongfu, Zhou Yuanhua and Huang Xiaodong, "Content Adaptive Spatial Error Concealment for Video Communication", IEEE Transactions on Consumer Electronics, Vol. 50, No. 1, pp. 335-341, 2004.

[10] D. Agrafiotis, D.R. Bull and C.N. Canagarajah, "Enhanced Error Concealment with Mode Selection", IEEE Transactions on Circuits and Systems for Video Technology, Vol. 16, No. 8, pp. 960-973, 2006.

[11] A. Criminisi, P. Perez and K. Toyama, "Object Removal by Examplar-based Image Inpainting”, Proceedings of IEEE Computer Society Conference on Computer Vision and Pattern Recognition, pp. 721-728, 2003.

[12] A. Criminisi, P. Perez and K. Toyama, "Region Filling and Object Removal by Exemplar-based Image Inpainting", IEEE Transactions on Image Processing, Vol. 13, No. 9, pp. 1200-1212, 2004.

[13] B.R. Li, Y. Qi and X.K. Shen, "An Image Inpainting Method", Proceedings of $9^{\text {th }}$ International Conference on Computer Aided Design and Computer Graphics, pp. 531536, 2005.

[14] C.Y. Chen, G.L. Wu and S.Y. Chien, "Hardware-Oriented Image Inpainting for Perceptual I-Frame Error Concealment", Proceedings of IEEE International Symposium on Circuits and Systems, pp. 836-839, 2008.

[15] L.W. Guan, Y.C Ching and Y.C. Shao, "Algorithm and Architecture Design of Image Inpainting Engine for Video Error Concealment Applications", IEEE Transactions on Circuits and Systems for Video Technology, Vol. 21, No. 6, pp. 792-803, 2011.

[16] Mounira Ebdelli, Olivier Le Meur and Christine Guillemot, "Analysis of Patch-based Similarity Metrics: Application to Denoising”, Proceedings of IEEE International Conference on Acoustics, Speech and Signal Processing, pp. 2070-2074, 2013.

[17] Mounira Ebdelli, Olivier Le Meur and Christine Guillemot, "Loss Concealment based on Video Inpainting for Robust Video Communication", Proceedings of $20^{\text {th }}$ European Signal Processing Conference, pp. 1910-1914, 2012.

[18] Hou Yu, Ma Ran, Li Panpan, An Ping, and Shi Nandi, "Hybrid Inter-frame Error Concealment Algorithm using Improved Image Inpainting", Journal of Computer-Aided Design and Computer Graphics, Vol. 28, No. 2, pp. 280287, 2016.

[19] Chengxing Li et al., "An Adaptive Error Concealment Algorithm based on Partition Model", Proceedings of Chinese Conference on Image and Graphics Technologies, pp. 35-43, 2016

[20] T.L. Lin, T.L. Ding, C.Y. Fan and W.C. Chen, "Error Concealment Algorithm based on Sparse Optimization", Multimedia Tools and Applications, Vol. 76, No. 1, pp. 397 413, 2017. 